\title{
An Expedition on Implementing the Cloud Data Centre using Shared Memory of Mobile Storage by Virtualization
}

\author{
Nazmus Sakib \\ Department of \\ Computer Science and \\ Engineering \\ Ahsanullah University of \\ Science and \\ Technology, Dhaka, \\ Bangladesh
}

\author{
Raihan Ahmed \\ Department of \\ Computer Science and \\ Engineering \\ Ahsanullah University of \\ Science and \\ Technology, Dhaka, \\ Bangladesh
}

\author{
Tanvir Ahmed \\ Department of \\ Computer Science and \\ Engineering \\ Ahsanullah University of \\ Science and \\ Technology, Dhaka, \\ Bangladesh
}

\author{
Fahad Bin Islam \\ Department of \\ Computer Science and \\ Engineering \\ Ahsanullah University of \\ Science and \\ Technology, Dhaka, \\ Bangladesh
}

\begin{abstract}
Cloud computing is now the biggest hotspot in modern era, which is spreading its impact in the technical site. For introducing a modern approach of cloud system, user's mobile devices are placed at the top and bottom layer of the cloud system while different virtual servers are present at the middle layer that forms the structure of three-tier technology. For implementing the established idea of the three tier cloud technology, a scenario with different users that has been demonstrated in the paper where users can upload and download data to/from the cloud server using their handheld devices whereas users' own device is also acting as cloud storage in behind scene. The result of the implementation of the three tier technology hashes out that the storage cost at the server can be roughly reduced by using a portion of users' mobile device storage as server storage. In consequences, a reduction of power consumption and maintenance cost happened at the cloud servers side due to less storage uses and increasing parallel processing in clients devices. Moreover, the solutions of some rising problems to the cloud organization can also be introduced after thoroughly with the analytics and statistics of the result of this expedition.
\end{abstract}

\section{Keywords}

Cloud, shared memory, virtualization, encryption, synchronization, RAID, security, Virtual Machine(VM), Media Access Control(MAC), Rivest-Shamir-Adleman (RSA), Advanced Encryption Standard (AES), Central Processing Unit(CPU)

\section{INTRODUCTION}

Cloud computing is now one of the rising topic which is not only used for technical purpose but also used for business model. As it is enriched with reliable data storage and fast access to the resource, it's demand is increasing among many organization for serving their customers with desired services. In the previous series of version, a cloud system consisting three-tier technology was proposed as following:

- First layer consists of user's mobile devices where user can initiate uploading or downloading request as well as other featured request to the cloud system.

- Second layer includes virtualized server performing encryption, decryption, mapping, mirroring and process request.

- Third layer again consist of users mobile devices but here users have no privilege over their mobile devices acting as shared storage of the server which ensures security of the data.

The three tier technology is implemented in this paper which demonstrates that some aspects of cloud computing can be improved [1], [2], [3], [4], [5].

The implementation follows the layer by layer steps of the proposed three tier technology. Each layer of the implementation characterizes the corresponding layer of the proposed three-tier technology.

- For implementation, the first layer of the proposed three-tier technology is provided with mobile devices that interacts with the cloud server based on the user's choice.

- Meanwhile the middle layer is enriched with virtualized servers such as process request, encryption-decryption, mapping, mirroring and device- data control.

- The top layer that is the storage of the user's mobile storage can be simply used and maintained as server storage by the middle layer.

The improvements are highlighted on storage capacity, security and power consumption in the implementation that reaches to a solution of some rising problems to the cloud organizations. By using user's mobile devices shared storage as server storage that is the third layer, results roughly reduction of storage needed at the server side by almost one third where server records one copy at server side and mirrors it on the third layer. As a result, cloud server can get rid of holding the mirrored data at server side allowing cloud server to consider less storage for holding only one copy of the user's data. Along with the reduction of storage needed, the power consumption is reduced at the server side as it needs to maintain almost one third of the cloud storage. Less storage maintenance results less CPU utilization as a result less power consumption. This paper follows in section two about the literature review then acknowledged idea on section three, after that approach of implementation and so on [6].

\section{LITERATURE REVIEW}

\subsection{Authentication}

Verifying a person's identity is required to allow access to confidential data or systems in computer science. Authentication is accepting proof of identity given by a credible person who has first-hand evidence that the identity is genuine also defines authentication by comparing the 
attributes of the object itself to what is known about objects of that origin. For example, a person who is the owner of house maybe allowed to enter his own house if he can prove himself as the owner. For ensuring safety of data in cloud system as well as ensuring authorized access in cloud a strong authentication process is must to authenticate the users.

In the implementation authentication is not focused though but along with established idea authentication is described and implemented with the app. When a user gets himself registered, he gets an id and password for further authentication purpose moreover; his mobile device's MAC is also recorded for the very authentication process. The log in the implementation is related with the authentication process as from the authentication records $\log$ is also created for identifying users and their data. After authentication, security is performed on the users data that is the next step for the three-tier technology [7], [8].

\subsection{Security}

Data security has consistently been a major issue in the cloud system environment, as the data is located in different places security in cloud system becomes particularly serious issue. Data security and privacy protection are becoming more important for the future development of cloud computing technology in government, industry, and business. Data security and privacy protection issues are relevant to both hardware and software in the cloud architecture. The more it becomes reliable and trustworthy the more users can get their minds off their data security.

In three-tier technology, data are provided with two layer security, where one is AES performed by the app that is supposed to be installed on every three-tier cloud system client's device and latter one is RSA performed by the server. In the implementation, security on users data is given once which is RSA by encryption-decryption visualized server of cloud network. After completion of providing security, mirroring on the particular encrypted data is performed by the cloud server [9], [10], [11], [12].

\subsection{Mirroring}

Mirroring is a process of system recovery where data is retrieved from mirror storage in case of data loss. Mirroring has different variations like RAID-0 , RAID-1 and RAID-5 .In this paper, the RAID-5 is needed and the idea of RAID-5 is generated from the storage cost as it is used to minimize the cost as low as possible. As RAID-5 data read transaction is very fast, which allows it to gain more importance to be used in three-tier technology. The performance of data storage is increased by using RAID-5. In addition, RAID-5 ensures the data reliability and availability by storing the data in different disks so that the data can be retrieved if one of them is lost or damaged. In the implementation, server performs mirroring on the data and stored is on the third layer mobile device storage. After mirroring, a mapping is performed to ensure efficiency and scalability of the server [13], [14], [15].

\subsection{Mapping}

In the implementation, the word mapping denotes finding out the available devices for storing user's data from the server side after giving two layer security on data and also for solving the download request of users by finding out the particular devices which holding the data of requesting user. Log is the most useful information in the mapping purpose because log holds all the information of users as well as which data is stored to whose mobile device. The information in the $\log$ is maintained updated after every transaction. To find the available devices to upload data and also to download data, mapping is used in the implementation with the help of log record [16], [17].

\subsection{Virtualization}

Virtualization is one of the main approach to distribute workload in the cloud system. By using virtualization technique many companies are able to lower their cost of the server so the demand of virtualization is getting higher day by day. Besides, for controlling several software a virtual software is used in a system which is Linux or windows in a single pc. Whenever there are huge request of the data the workload is distributed by virtualization using virtualized servers. In a result, the virtualized servers carry out the request of the users.

There are three types of virtualization technique. They are server virtualization, desktop-client virtualization and storage virtualization. In server virtualization, workload is distributed in the cloud which is maximizing the resource utilization in the server and minimizing the cost of the server. Desktop client virtualization helps to improve the security. Storage virtualization has three approaches which have Direct Attached Storage (DAS), Network Attached Storage (NAS) and SAN (Storage Area Network). Each of the storage has different technique to store data and maintain security [18], [19], [20].

\section{ACKNOWLEDGED IDEA}

An acknowledged idea for cloud system that is three tier technology where the first layer and third layer are consist of user's mobile device and the middle layer is the virtualized server. The first layer mobile device is used to access the cloud featuring downloading, uploading data and other request to the cloud server, importantly the third layer is used as the data storage which builds the data center of the cloud system. The middle layer is consisted of deferent virtualized servers performing the core tasks of the cloud system which are: encryption decryption, mirroring, mapping, process request etc. For accessing the cloud an app must be installed on the mobile device which is used for the authentication purpose. Whenever the app is installed on the mobile device a portion of the mobile storage is separated and used as the cloud storage [1], [21], [22]. 


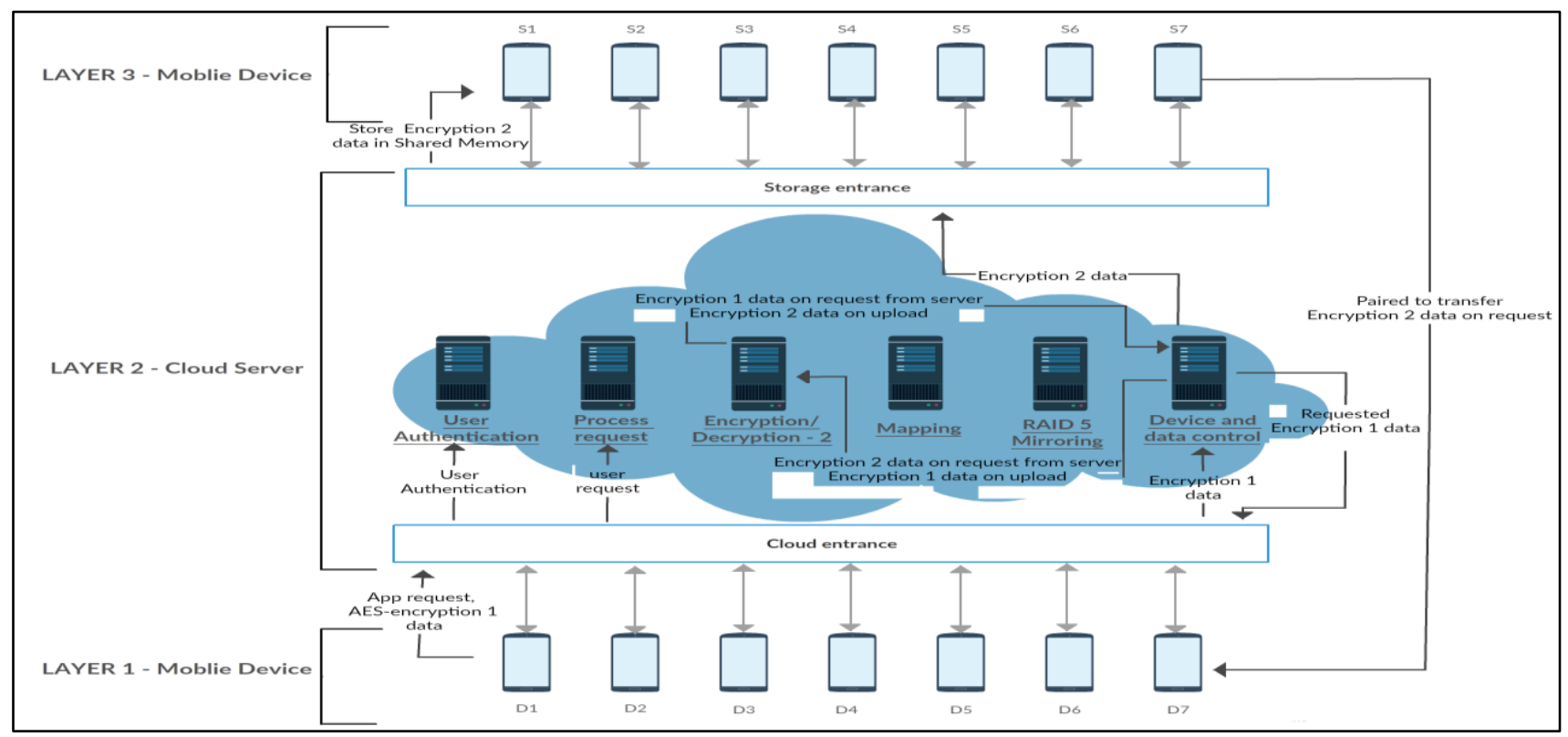

Fig 1: Three Tier Technology

The flowchart of the acknowledged idea illustrates the downloading and uploading process of the three tier technology. For example a device D1, to upload data user authentication is checked by the server first, then it is checked whether user wants to upload or download data.

If D1 chooses to upload then encryption-1 that is AES perform by the app on the data then it is sent to the server where server performs encryption 2 that is RSA on the received data then mapping and mirroring on the received encrypted data. Meanwhile suppose S3, S4, S5 and S7 are available layer 3 devices among which S3 and S4 are selected as the best devices for mirroring based on available storage, distance from the server etc. Finally data is stored on the selected best two devices of layer 3 as well as in the server side itself and the $\log$ file is updated accordingly [1].

On the other hand if D1 attempts to download file, a request from the device is sent to the server for specific data and the server response by mapping the nearest best device with the help of the $\log$ file. Suppose it succeeds finding the available layer 3 device that is S3 then a pair is made between the requesting device D1 and the layer 3 device S3 to directly download the data to the requesting device. Here decryption 2 and decryption 1 is performed by the app to display the data. If the server fails to find available layer 3 device then server side data is used to serve the request by performing decryption 2 on the data at the server side then it is transferred to the requesting device D1 where decryption 1 is performed before displaying the data. This idea introduces a two layer security on the user data, maximum utilization of the mobile shared memory, importantly reduces the storage cost for the server along with power consumption and maintenance complexity [1].

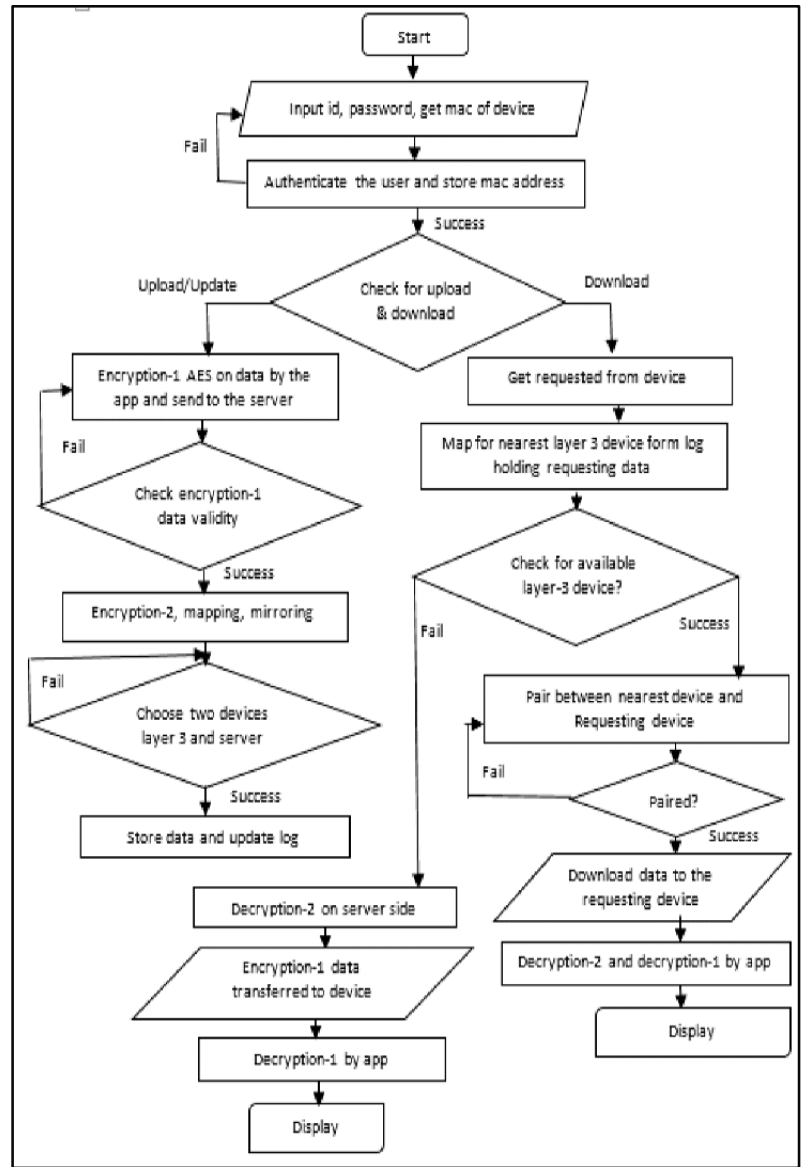

Fig 2: flow diagram to upload and download data

4. APPROACH OF IMPLEMENTATION

In this paper, the noble approach of using cloud system, the 3tier technology has been implemented with the help of the matlab Simulink which follows a two-step transformation of the cloud system. Here, in the first step, the whole cloud system is segmented into three layers where bottom layer that 
is the first layer, is the sending layer while the top layer that is the third layer, is the receiving layer and the whole process is organized by the monitoring of the middle layer. On the next step, the middle layer is also fragmented to comprise the different virtual servers to perform encryption, decryption, device and data control, process request, mapping, mirroring. Instead of many users at the first layer, two users are consider to represent the multiple user environment of the cloud server sending different requests to the cloud server. The third layer, consists only the data that has been uploaded from the first layer and that can be maintained by the middle layer.

In three tier technology, the first and third layer are user mobile device but the difference between the usages of these two layers' mobile device has been hashed out in this implementation which recites that the first layer mobile device enables users to access the cloud anytime from anywhere and the third layer mobile devices serve the need of storage for the cloud server.

The flexible and secured access of the stored data at the third layer are being monitored and handled simultaneously by the middle layer that is the virtualized servers performing different specific tasks such as device and data control, mirroring, mapping, process request, encryption-decryption that has been introduced in the implementation.

In the purpose of serving cloud service, each user is provided with a maximum limit of usable cloud storage that can be introduced by the third layer mobile devices' storage. Here the whole available third layer storage is segmented according to the number of the users with a maximum limit where the last value of each segmented storage for each users indicates how much spaces have been used by a particular user. Suppose that a user gets a segmented space of 51 unit where the last 51th unit contains a number implying the number of space used by the following user. If 51th unit consists a value of 15 then the total free space would be $y$.

$$
\begin{aligned}
& y=\int_{x=0}^{x=1} \frac{(z-1)-y}{z} * 100 \% \\
& =\frac{(51-1)-15}{51} * 100 \% \\
& =68.63 \% \text { free space }
\end{aligned}
$$

Where, $\mathrm{z}=$ total space, $\mathrm{y}=$ used space and $\mathrm{z}[0,1]$ implies $\mathrm{z}=$ 0 for an empty segment and $\mathrm{x}=1$ for a full segment.

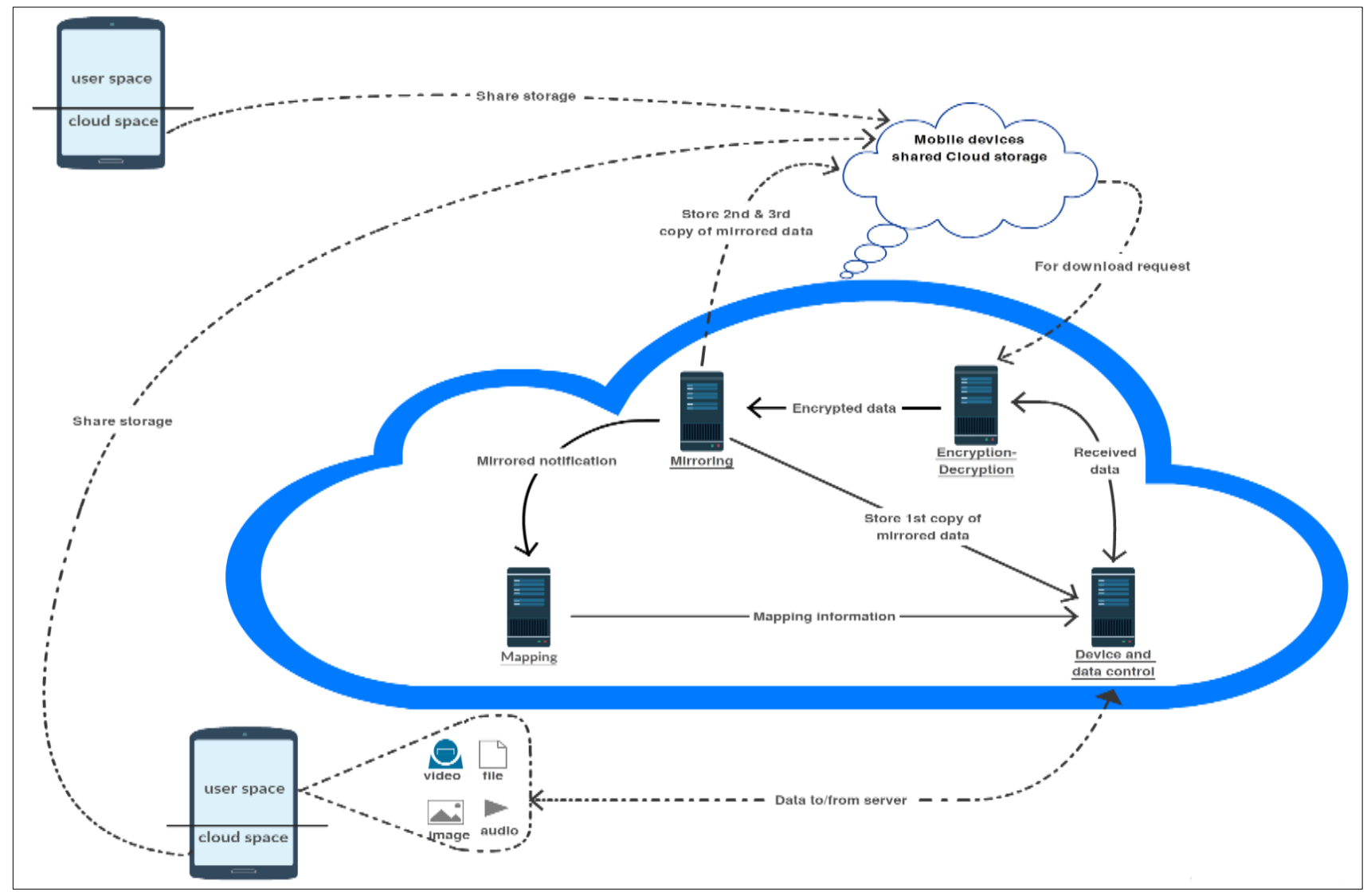

Fig 3: Implementation approach of 3-tier technology

For a user of first layer, users that are actively connected with the cloud server can request for upload and download data simultaneously to the server. To upload a data to the cloud server a user firstly selects the data by user input which results the data to be sent to the server directly. Hence, the device and data control virtual machine holding the users' data for further processing. Later, server performs encryption on the user's data using RSA algorithm providing high security on the user data.
Hereafter the encrypted data from the encryption decryption virtual machine is fed into mirroring virtual machine that mirrors the $1^{\text {st }}$ copy of the encrypted data at server side for providing further flexibility and scalability for the cloud server. And also, the $2^{\text {nd }}$ and $3^{\text {rd }}$ copy of encrypted data is pushed to the third layer that is client's mobile devices shared cloud storage. Meanwhile, the cloud server activate the user that is interacting with the server, which enables to select the segmented memory layer 3 storage block to upload the 
specific user data, results the mirroring of the user data on third layer is performed. In this procedure, the specific user data is uploaded on the third layer as mirrored data to the allocated user storage, moreover data is also stored on the server side providing server scalability and reliability in case of layer 3 storage failure.

Now for a user to request for a pointed out data that is stored on the cloud server, the choice is sent for requesting a download to the server. Thereafter, server serves the requesting user by enabling layer 3 storage specific user block and selecting the pop index value of the desired requested data, resulting the requested data of a particular user is selected from that specific user's segmented storage then it is fetched from the layer 3 storage block. In this case, the data pulled data of layer 3 storage is in encrypted form that is passed to the encryption decryption block for performing decryption on the requested data. In the meanwhile, download request activates to pass the permission to decrypt the data. Upon receiving the permission from the download request, the encryption decryption block decrypts the requested data and passes the decrypted data to the process download request block which routes the decrypted data to the requesting. Later on the data is displayed to the requesting user where the data will be readable.

In the following way, a user can request to upload data to the server, where the server performs encryption, mapping, mirroring, processing upload request and stores the encrypted data on the third layer that is users mobile device consisting segmented storage for individual users. In addition, server can inform the user about the usage of the allocated layer 3 storage for a particular user using the value of last unit of the segmented storage. After uploading user data, a user can request to the server for a specific data which server handles by fetching the requesting data from layer 3 storage. After performing decryption on the fetched data from the layer 3 storage, server then serves the user with the requested decrypted data based on the request made by the user.

\section{RESULT ANALYSIS}

The implementation of the three-tier technology is followed by blind test with random data retrieval. For a user, if it is considered processing of integer value within the network then different bit length produces different processing time.

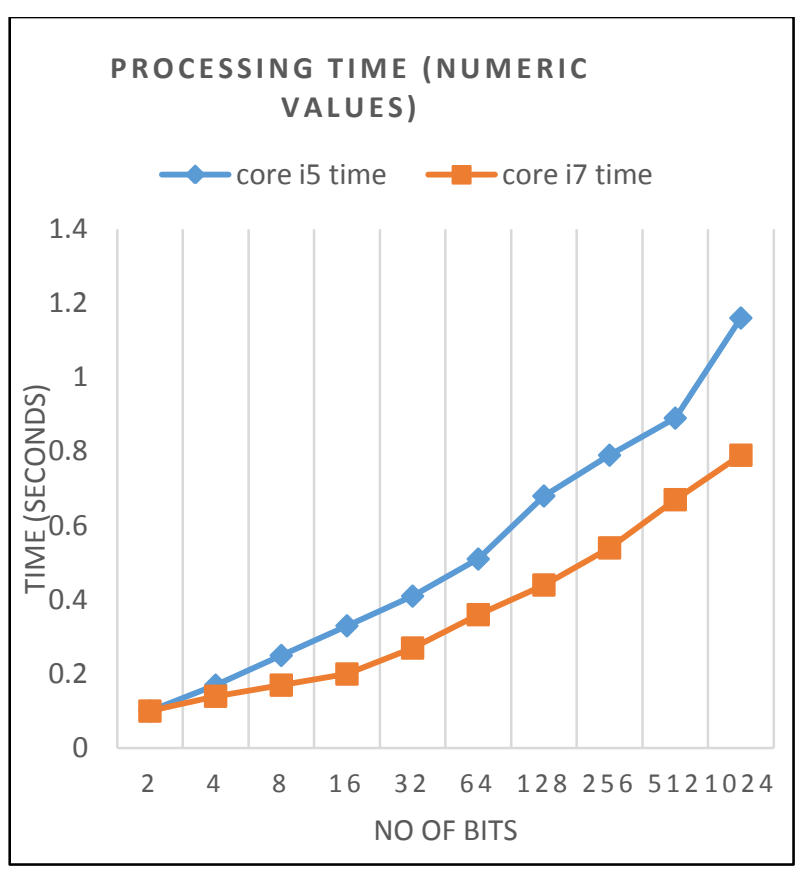

Fig 4: Processing time for text

In core i5 processor to process numeric values, the blind test shows that the processing time with the increasing bits of the input increases. When the no of bits are increased from one bit to ten bits then the processing time is increased to 1.16 seconds.

In core $\mathrm{i} 7$ processor to process numeric values, the blind test shows that the processing time with the increasing bits of the input increases but in this case the increase is quite less. When the no of bits are increased from one bit to ten bits then the processing time is increased to 0.79 seconds.

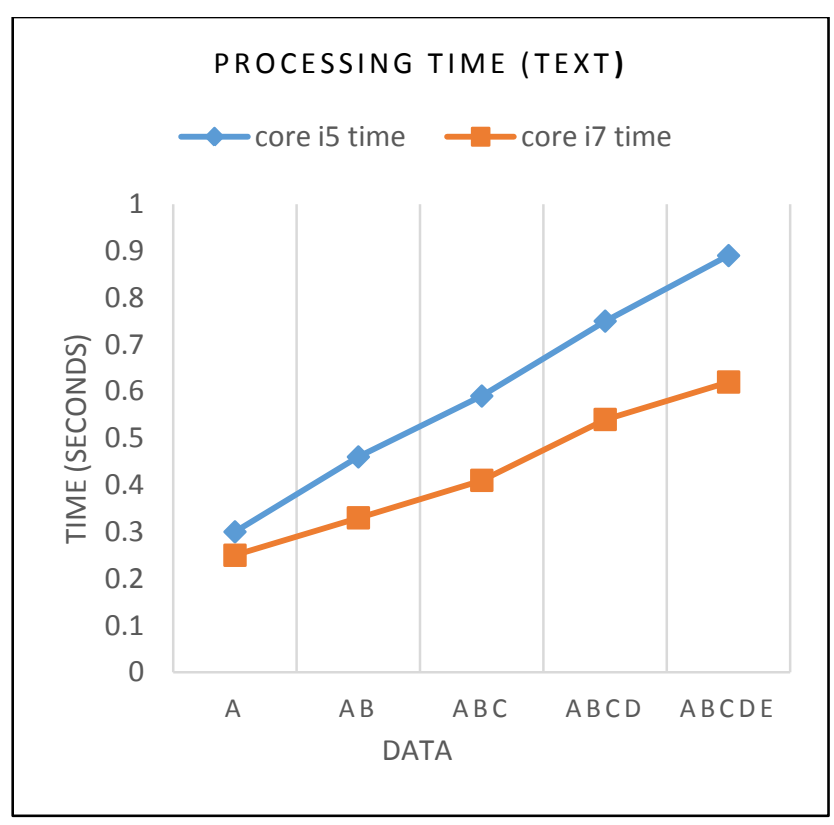

Fig 5: Processing time for numeric values

On the other hand, in core i5 processor to process text, the blind test shows that the processing time with the increasing complexity of input data increases. The highest complexity 'ABCDE' takes 0.89 seconds to process. 
In core i7 processor to process text, the blind test shows that the processing time with the increasing complexity of input data increases but it take less time comparatively to the core i5. The highest complexity ' $\mathrm{ABCDE}$ ' takes 0.62 seconds to process. Therefore, the blind test demonstrates that the processing time is machine dependent [23], [24], [25], [26].

The three-tier technology stated that the cloud server receives a data from the user and performs mirroring on the data to produce two extra copy that can be stored on the third layer, which is user's mobile devices shared memory along with one copy is kept at server side. If the server were to store the three copies at server side then the storage needed at the server side would be huge, resulting a high storage cost, power consumption and maintenance cost for cloud server. By relying on the three-tier technology, server can store two of the three copies on the users mobile devices shared memory and only one copy at server side instead of storing all the three copies at the server side which ensures that the storage needed at server side can be roughly reduced by one third. Indicating that the cloud server needs to bear only the cost of storage to store one copy of the user data, moreover cloud server would be highly encouraged to serve their users with increasing amount of storage at a low price. So both the cloud server and the users would be highly benefitted by this policy [27], [28]. If the storage need by a usual cloud server is $\mathrm{x}$ and the storage needed by three-tier technology is $y$ then it can be expressed with the following equation

$$
y=F(x)=\frac{1}{3} * \frac{x}{2} * \int\left(\frac{\ln (x)}{\log (x)}\right)
$$

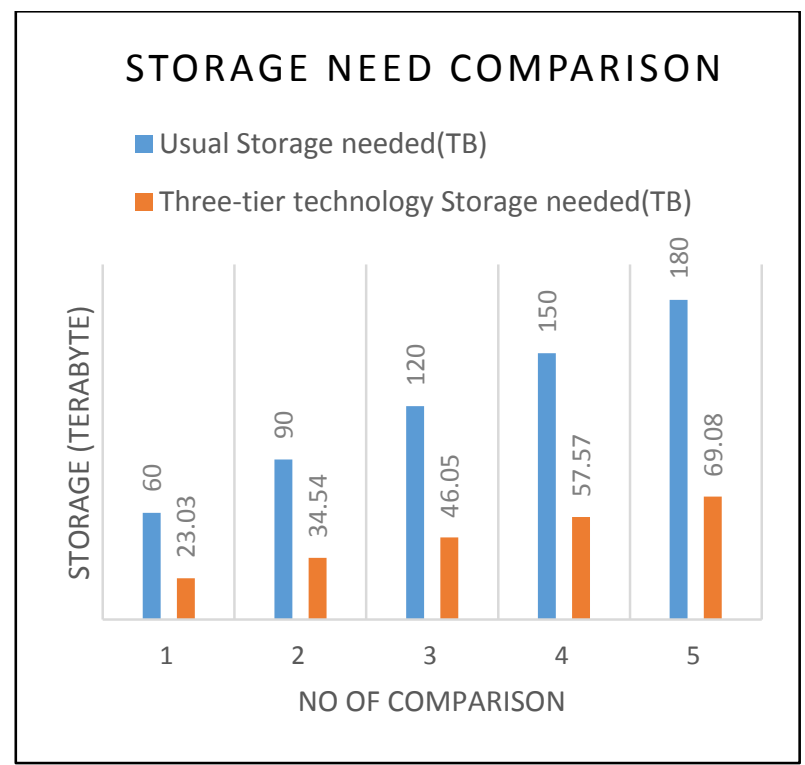

Fig 6: Storage cost comparison after implementation

Here, $\int\left(\frac{\ln (x)}{\log (x)}\right)$ gives a fluctuation ratio based on different hardware configuration that is multiplied by $\frac{x}{2}$ then the one third of the storage is the expected storage. Storage needed comparison proves that the storage needed by the three-tier technology is almost one third of the usual storage needed by a cloud server. With the storage need at the serve side decreases, not only the storage cost but also the power consumption by the server side goes down at large, as lesser storage than the previous storage is maintained.

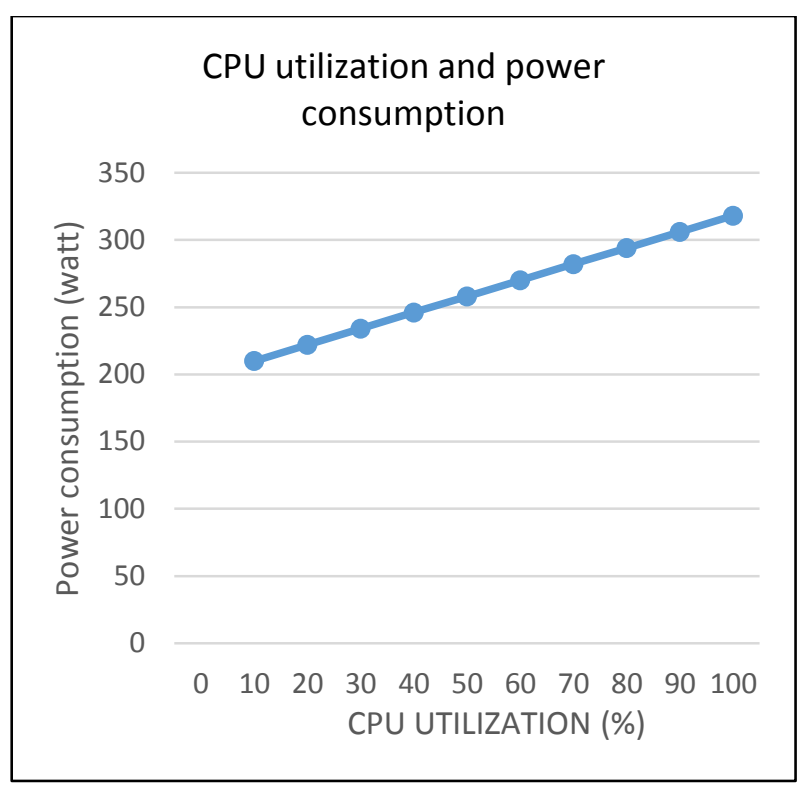

Fig 7: Utilization and power consumption [6].

Figure Utilization and power consumption graph shows that power consumption of a CPU (central processing unit) increase linearly with the increase of CPU utilization.

$$
P(n)=P_{\text {idle }}+\left(P_{\max }-P_{\text {idle }}\right) * \frac{n}{100}
$$

With the help of the above equation [6], power consumption $P(n)$ can be calculated where, $P_{\text {idle }}$ is power consumed by the server when it remains idle is the lowest cpu utilization, $P_{b}$ is the power consumed by the server when its utilization is $\mathrm{n}(\%)$.

Another relaxing situation is created with the help of parallelism that is achieved by using the processor of the cloud user's handheld mobile devices. With increasing number of users for the cloud system, three-tier technology, the number of processors that are involved with the cloud system also increases. As a result the parallelism of the cloud system, the three-tier technology, increases as the processors of the clients handheld device also plays a supportive role in the cloud system. With the help of Amdahl's Law the parallelism can be expressed.

$$
\int_{N=1}^{N=\infty} \frac{d}{d p} \frac{1}{(1-p)+\left(\frac{P}{N}\right)}
$$

Here, the equation defines the maximum speedup achieved where $\mathrm{p}$ indicates the parallelism of the system and $\mathrm{N}$ defines the number of processors. $\mathrm{N}$ can be a value infinity, in this case the maximum speed up would be $\frac{1}{(1-p)}$. It shows that the three-tier technology can cope up with preferred speed while increasing users [29].

Now, in the three-tier technology it is shown that server would need to maintain almost one third of the storage needed than usual which allows server to maintain less storage resulting less pressure on the server led to low utilization. In the consequences, the power consumption would be low. The mentioned analysis shows that, the three-tier technology would be a good option for a cloud server to rely on. Along with storage cost and power consumption reduction the maintenance would be easy resulting the whole cloud server having a better flexibility, scalability and reliability. 


\section{CONCLUSION}

Cloud computing is now the trending technology which is revolving rapidly with the changing need of the customer and the serving approach of the different established organization through which cloud computing is introducing its newest forms of accretion resulting the desired services to appear as more friendly to the customer uses. The implementation of the proposed 3-tier technology can be considered as one of the developed form of cloud management that shows the new path of combining uses of cloud server with users' mobile devices enhancing the reduction of storage need at the server side which result low power consumption along with cost deduction to ensure the organization to sit back with the ensured positive outcome. One the other hand, customers of the cloud service can remain in a safe mind set about their data security, manageability and reliability on cloud server. Above all, the 3-tier technology offers the optimized use of the users unused mobile device storage by adding them as the part of the cloud server storage which helps server to serve each user with an increased amount of storage so that the cloud server and the customers can be benefitted with the approach of using this acknowledged three tier technology.

\section{FUTURE WORK}

In future, we are focusing on building this particular app for this cloud system with updated security mechanism and optimization of data sharing in an organized way to process and share data more faster than before. Moreover, faster file sharing using direct pair between two devices within the cloud would be introduced by this app.

\section{REFERENCES}

[1] N. Sakib, R. Ahmed, T. Ahmed, B. Islam and B. Das, "A Proposal on Cloud Based Data Centre Using Shared Memory of Mobile Storage by Virtualization," International Journal of Applied Information System, vol. 11, no. 12, pp. 1-6, 2016.

[2] E. Gorelik, "Cloud Computing Models," Massachusetts Institute of Technology, USA, 2013.

[3] K. V. S. R. a. M. Q. J. SRINIVAS, "CLOUD COMPUTING BASICS," International Journal of Advanced Research in Computer and Communication Engineering, vol. 1, no. 5, pp. 1-3, 2012.

[4] K. W. E. G. S. F. S. D. A. M. N. R. A. W. P. S. F. M. J. L. G. C. B. L. K. H. V. K. a. M. M. A. Holt, "Cloud Computing Takes Off," MORGAN STANLEY RESEARCH Global, 2011.

[5] D. G. R. E. F.-M. a. E. B. F. K. Hashizume, "An analysis of security issues for cloud computing," Journal of Internet Services and Applications, vol. 4, no. 5, p. 1, 2013.

[6] "InfoQ," [Online]. Available: https://www.infoq.com/articles/power-consumptionservers. [Accessed 10 january 2017].

[7] "TechTarget," [Online]. Available: http://searchsecurity.techtarget.com/definition/authentica tion-server. [Accessed 20 january 2017].

[8] M. Vellon. [Online]. Available: http://www.networkworld.com/article/2194263/techprimers/authentication-in-the-cloud.html. [Accessed 5 january 2017].
[9] J. Z. Y. X. a. G. Z. YunchuanSun, "Data Security and Privacy in Cloud Computing," International Journal of Distributed Sensor Networks, 2014

[10] D. G. R. E. F.-M. a. E. B. F. K. Hashizume, "An analysis of security issues for cloud computing," Journal of Internet Services and Applications, vol. 4, no. 5, pp. 1-5, 2013.

[11] U. N. a. V. Kotak, "Security Issues with Implementation of RSA and Proposed Dual Security Algorithm for Cloud Computing," IOSR Journal of Electronics and Communication Engineering (IOSR-JECE), vol. 9, no. 1, pp. 1-5, 2014

[12] P. D. a. P. M. Pitchaiah, "Implementation of Advanced Encryption Standard Algorithm," International Journal of Scientific \& Engineering Research, vol. 3, no. 3, pp. 1-5, 2012.

[13] i. nsk. [Online]. Available: http://blog.nskinc.com/ITServices-Boston/bid/77545/When-to-Use-Mirroring-asa-Data-Recovery-Solution. [Accessed 28 july 2016].

[14] H. J. a. K. Hwang, "Stripped mirroring RAID architecture," Journal of Systems Architecture, vol. 46, no. $543 \pm 550$, pp. $1-6,2000$.

[15] "Prepressure," [Online]. Available: https://www.prepressure.com/library/technology/raid. [Accessed january 2017].

[16] "DriveHQ," [Online]. Available: https://www.drivehq.com/mapdrive/. [Accessed 25 january 2017].

[17] "Microsoft," [Online]. Available: https://msdn.microsoft.com/en-us/library/dd430340.aspx. [Accessed 27 january 2017].

[18] K. Khajehei, "Role of virtualization in cloud computing," International Journal of Advance Research in Computer Science and Management Studies, vol. 2, no. 4, pp. 1-8, 2014.

[19] S. N. J. S. W. A. a. A. G. M. Liaqata, "Virtual Machine Migration Enabled Cloud Resource Management: A Challenging Task," University of Malaya, kualalampur and Jabalpur, 2016.

[20] D. M. a. K. P, "A Study On Virtualization Techniques And Challenges In Cloud Computing," INTERNATIONAL JOURNAL OF SCIENTIFIC \& TECHNOLOGY RESEARCH, vol. 3, no. 11, pp. 1-3, 2014.

[21] "WdSupport," [Online]. Available: http://support.wdc.com/knowledgebase/answer.aspx?ID= 10624. [Accessed 23 december 2016].

[22] M. S. a. A. Shukla, "Implementation of Location based Services in Android," IJCSI International Journal of Computer Science, vol. 9, no. 1, pp. 1-4, 2012.

[23] "Apica," [Online]. Available: https://www.apicasystem.com/blog/cloud-performancetesting-cloud-based-applications/. [Accessed 22 january 2017].

[24] "Techtarget," [Online]. Available: http://searchsoftwarequality.techtarget.com/feature/Perfo rmance-testing-in-the-cloud. [Accessed 12 january 2017] 
[25] H. F. M. Satish Srirama, "REuse and Migration of legacy applications to Interoperable Cloud," 2012.

[26] P. J. Eckstein, CALCULATIONS," 2007.

[27] "VmWare,"

[Online].

STORAGE https://www.vmware.com/cloud-services/pricingguide.html. [Accessed 22 november 2016].
[28] "Rackspace," [Online]. Available: https://www.rackspace.com/cloud/servers/pricing. [Accessed 29 november 2016].

[29] Techopedia. [Online]. Available: https://www.techopedia.com/definition/17035/amdahlslaw. 